\title{
Comparison of Phenotypic MRSA Detection Methods with mecA gene PCR in a Tertiary Care Centre in India
}

\author{
Dani Kochuplapparambil Thampi ${ }^{1}$, Nizamuddin Mundangalam², Shoba Kurian Pulikottil ${ }^{3}$, Netto Jacob ${ }^{4}$ \\ ${ }^{1}$ Specialist, Department of Microbiology, Aster MIMS Hospital, Calicut, Kerala, India. \\ ${ }^{2}$ Associate Professor, Department of Microbiology, Government Medical College, Kollam, Kerala, India. \\ ${ }^{3} \mathrm{HOD}$, Department of Microbiology, Government Medical College, Kottayam, Kerala, India. \\ ${ }^{4}$ Specialist, Department of Microbiology, Bharat Hospital, Kottayam, Kerala, India.
}

\section{ABSTRACT}

\section{BACKGROUND}

Exact laboratory confirmation of MRSA isolates is crucial for infection management, control \& prevention of MRSA. In resource restrained centers, the unavailability of PCR leads to dependence on other phenotypic methods.

\section{METHODS}

The study was conducted in the Department of Microbiology, Government Medical College, Kottayam, for a period of one year from July 2015. The study design was Analytical Diagnostic Test Evaluation. The study was designed at comparing different phenotypic methods in their sensitivity, specificity, positive predictive value, negative predictive value and accuracy, keeping mec-A gene detection by PCR as the gold standard. The following six phenotypic methods were performed on Staphylococcus aureus isolates- Cefoxitin Disc Diffusion (CDD), Oxacillin Disc Diffusion (ODD), Oxacillin Screen Agar (OSA), Oxacillin E-Strip (ES), Chromogenic agar medium (ChromID® MRSA SMART-biomerieux) and VITEK-2 system (biomerieux). These were then compared with the gold standard method- mecA gene detection by PCR.

\section{RESULTS}

Cefoxitin disc diffusion test and VITEK 2 system showed results in agreement with PCR (Sensitivity and specificity 100\%). This was followed by Chrome agar (98.36\%, 95.79\%), Oxacillin screen agar (95.08\%, 98.95\%), Oxacillin E-test (95.08\%, 97.89\%) \& Oxacillin disc diffusion test $(81.96 \%, 97.89)$.

\section{CONCLUSIONS}

Cefoxitin disc diffusion test which shows total agreement with PCR is a good surrogate marker for detecting methicillin resistance. However, VITEK-2 system and chromogenic agar media are valuable as they are useful in early detection and screening of MRSA.

\section{KEY WORDS}

MRSA, Cefoxitin Disc Diffusion, Oxacillin, Phenotypic, mec-A

\author{
Corresponding Author: \\ Dr. Dani Kochuplapparambil Thampi \\ Vakathanam, P. O., Kottayam, \\ Kerala, India. \\ E-mail:danikthampi89@gmail.com
}

DOI: $10.14260 /$ jemds/2019/610

Financial or Other Competing Interests: None.

How to Cite This Article:

Thampi DK, Mundangalam N, Pulikottil SK, et al. Comparison of phenotypic MRSA detection methods with mecA gene PCR in a tertiary care centre in India. J. Evolution Med. Dent. Sci. 2019;8(36):2813-2817, DOI: $10.14260 / \mathrm{jemds} / 2019 / 610$

Submission 06-07-2019, Peer Review 24-08-2019, Acceptance 30-08-2019, Published 09-09-2019. 


\section{BACKGROUND}

The first reports of a Staphylococcus aureus strain that was resistant to methicillin was published in 1961. Outbreak of infections caused by Methicillin Resistant Staphylococcus aureus (MRSA) strains were reported in hospitals in United States in 1970s and by 1980s. These strains swept the globe leading to the worldwide pandemic of MRSA in hospitals that continues to the present time.[1]

The main mechanism of methicillin resistance in Staphylococcus aureus is through the expression of a foreign PBP (Penicillin Binding Protein), PBP2a. Resistance to methicillin confers resistance to all $\beta$-lactam agents, including cephalosporins.[2] External factors such as, salt concentration, $\mathrm{pH}$, medium composition, osmolarity and temperature are exploited in the clinical laboratory to enhance the detection of strains exhibiting heterogeneous methicillin resistance.[3]

Nosocomial bacteraemia with Staphylococcus aureus occurs secondary to a staphylococcal infection elsewhere or by the direct access of the organism into the bloodstream via catheters and other indwelling devices. Mortality related to Staphylococcus aureus BSI is high, ranging from 20\% to $30 \%$ of the cases, and varies as a function of underlying conditions. ${ }^{[4]}$ Staphylococcus aureus became one of the most frequent pathogens responsible for nosocomial pneumonia of which more than $55 \%$ is MRSA.[5]

Osteomyelitis due to Staphylococcus aureus most often occurs as a complication of local infection via direct extension, although haematogenous osteomyelitis may occur. Haematogenous osteomyelitis usually occurs in young children, but osteomyelitis of the vertebral column accounts for $60 \%$ of such infections in adults.[6]

The patient's endogenous skin flora, with gram-positive organisms, in general, and staphylococcal species, in particular, are the predominant cause of incision infections of clean surgical procedures. As reported by the National Healthcare Safety Network (NHSN), January 2006 to October 2007 (USA), $30 \%$ of surgical site infections are due to Staphylococcus aureus.

Treatment for MRSA differs from that of Methicillin susceptible Staphylococcus aureus (MSSA). So laboratory confirmation of Staphylococcus aureus isolates and antibiotic susceptibility testing became crucial in infection management, control and prevention of MRSA.

Different studies in India show varying proportion of MRSA ranging from $29.10 \%$ to $70.70 \%$.[7] Various factors influences occurrence of MRSA in different hospitals and adds to morbidity and mortality. ${ }^{[8,9]}$

Phenotypic tests for detection of MRSA mainly used in various laboratories include Cefoxitin Disc Diffusion (CDD), Oxacillin Disc Diffusion (ODD), Oxacillin Screen Agar test (OSA), Oxacillin E-strip (ES) Chromogenic agar medium and VITEK-2 system.

This study was conducted to compare these six phenotypic diagnostic methods for detection of MRSA isolates compared with gold standard- mecA gene detection by PCR to find out most suited method to use in resource restricted centres.

Also, the prevalence of MRSA and the associated antibiotic susceptibility profile needs to be ascertained.

\section{METHODS}

Analytical diagnostic test evaluation, by comparing the phenotypic tests for detection of MRSA strains with mecA PCR is the study design used.

The study was conducted for a period of one year from July 2015 at Government Medical College, Kottayam, Kerala, India. Study was started after obtaining clearance from the Institutional Ethics Committee. 156 isolates of Staphylococcus aureus cultured from clinical samples were included in this study.

Sample size was calculated as per the formula-

$\mathrm{N}=\frac{\mathrm{a}}{\text { prevalence }}$ where,

' $\mathrm{N}$ ' is actual sample size.

' $a$ ' is expected sensitivity of the test and denominator is the Prevalence of MRSA in South India in previous studies (30\%)

$\mathrm{a}=\frac{\mathrm{S}(1-\mathrm{S})}{\mathrm{D}^{2}}$ where,

' $\mathrm{s}$ ' is the sensitivity value of the test with lowest sensitivity from previous studies (97\%; ' $D$ ' is allowable deviation $(2.5 \%)$. Each isolate was identified as Staphylococcus aureus by gram stain, culture and relevant biochemical tests.

\section{Phenotypic Methods for MRSA Detection[10]}

Following tests were done with all the 156 isolates to characterise MRSA and MSSA (Methicillin Sensitive Staphylococcus aureus)

\section{Cefoxitin Disc Diffusion Test (CDD)}

A 0.5 McFarland standard suspension of the isolate was made, and lawn culture was done on MHA (Mueller Hinton Agar) plate. All the isolates were subjected to cefoxitin disc diffusion test using a $30 \mu \mathrm{g}$ disc. Plates were incubated at $37^{\circ} \mathrm{C}$ overnight and zone diameter was measured. An inhibition zone diameter of $\leq 21 \mathrm{~mm}$ was reported as methicillin resistant and a diameter of $\geq 22 \mathrm{~mm}$ was considered as methicillin sensitive.

\section{Oxacillin Disc Diffusion Method (ODD)}

The Oxacillin disc $(1 \mu \mathrm{g})$ diffusion method was carried out on Mueller-Hinton agar (MHA) which was supplemented with $2 \% \mathrm{NaCl}$ to detect MRSA.

The isolates were considered as resistant when the diameter of inhibition was $\leq 10 \mathrm{~mm}$, as intermediately resistant when the diameter was $11-12 \mathrm{~mm}$ and as sensitive when the diameter was $\geq 13 \mathrm{~mm}$.

\section{Oxacillin Screen Agar Test (OSA)}

A bacterial inoculum of each strain was made, and turbidity was adjusted to $0.5 \mathrm{McF}$ arland. One drop of this suspension was spot inoculated onto Mueller-Hinton agar containing $4 \%$ $\mathrm{NaCl}$ and $6 \mu \mathrm{g} / \mathrm{ml}$ oxacillin. Plates were incubated at 35을 24 hours. Any strain showing growth on the plate containing oxacillin was considered to be resistant to methicillin. 


\section{Oxacillin E-Strip (ES)}

When E-strip is applied on to agar surface there is immediate transfer of the preformed antibiotic gradient into the agar matrix. After incubation, whereby bacterial growth becomes visible, a symmetrical inhibition ellipse centered along the strip is seen. The MIC value is read from the scale in $\mu \mathrm{g} / \mathrm{ml}$ where the pointed end of the ellipse intersects the strip. Isolates with MIC $>6 \mu \mathrm{g} / \mathrm{ml}$ were interpreted as MRSA.

\section{Chromogenic Agar Medium}

'ChromID® MRSA SMART' (biomerieux) agar is a ready to use chromogenic medium for the screening of MRSA.

\section{VITEK- 2 System}

Susceptibility to anti-staphylococcal agents- Vancomycin, Teicoplanin, Linezolid, Daptomycin, Rifampicin, and Tigecycline were tested using VITEK-2 compact system using GP-628 AST cards. This automated system determines the minimum inhibitory concentrations (MIC) of different antibiotics against each isolate and interpretation is done by the system's software based on CLSI guidelines.

The detection of MRSA in this system is by a combination of cefoxitin screen test and oxacillin MIC measurement. The system's inbuilt software will interpret the isolate as methicillin susceptible or resistant based on the results of these two tests.

\section{PCR for Detection of mecA gene}

MRSA and MSSA isolates were confirmed by mecA PCR which is the gold standard. Polymerase Chain Reaction (PCR) assay for the detection of mecA gene - the gold standard test - was carried out at the Cholera and Biofilm Laboratory, Rajiv Gandhi Centre for Biotechnology, Trivandrum, Kerala, India.

$\begin{array}{ll}\text { Master-Mix of a Single Reaction } & \\ \text { Buffer } & 2 \mathrm{uL} \\ \mathrm{MgCl}_{2} & 2 \mathrm{uL} \\ \mathrm{dNTPs} & 1.5 \mathrm{uL} \\ \mathrm{MecA} \text { F Primer } & 0.5 \mathrm{uL} \\ \text { MecA R Primer } & 0.5 \mathrm{uL} \\ \text { Taq polymerase } & 0.3 \mathrm{uL} \\ \text { Template DNA } & 1 \mathrm{uL} \\ \text { MilliQ } \mathrm{H}_{2} \mathrm{O} & 12.2 \mathrm{uL} \\ \text { The volume of one reaction is } & 20 \mathrm{uL} .\end{array}$

The primer used was supplied by Sigma Genosys, UK. The primers used were mecA F primer (5'AAAATCGATGGTAAAGGTTGGC-3') which corresponds to nucleotides 1282 to 1303 and mecA R primer (5'AGTTCTGCAGTACCGGATTTGC-3') which is complementary to nucleotides 1581 to 1598 of the coding frame. This yields a product of $533 \mathrm{bp}$ (base pair) size.

When the PCR was completed, $10 \mu \mathrm{L}$ of PCR products were withdrawn and mixed with a loading dye. These are then electrophoresed on $1 \%$ agarose gel at $70 \mathrm{~V} / \mathrm{cm}$ for 1 hour, stained using $1 \%$ ethidium bromide and viewed on GENE-DOC (UV-transilluminator). A 100 bp ladder DNA is used as the molecular weight marker against which the products are compared.

All the necessary standard precautions and steps to prevent contamination of PCR were taken.
The data was numerically coded and was entered in Microsoft Excel spreadsheet. Further analysis was done using the software SPSS 16 and tables and charts were created using Excel. Statistical significance was noted when p-values were less than 0.05 .

\section{RESULTS}

Out of 156 Staphylococci isolates, 61 were confirmed as MRSA by mecA gene amplification (PCR). 95 isolates negative for mecA gene were confirmed as MSSA.

\begin{tabular}{|c|c|c|}
\hline PCR & Number & Percentage \\
\hline Positive (True MRSA) & 61 & $39.10 \%$ \\
\hline Negative (True MSSA) & 95 & $60.90 \%$ \\
\hline Total & 156 & $100 \%$ \\
\hline \multicolumn{2}{|c|}{ Table 1. Result of mecA gene Amplification Study }
\end{tabular}
(Polymerase Chain Reaction Assay)

\begin{tabular}{|c|c|c|c|c|}
\hline \multirow{2}{*}{ Test } & \multicolumn{2}{|c|}{ MRSA } & \multicolumn{2}{c|}{ MSSA } \\
\cline { 2 - 5 } & $\begin{array}{c}\text { Number of } \\
\text { Isolates } \\
\text { Detected as } \\
\text { MRSA }\end{array}$ & $\begin{array}{c}\text { Number of } \\
\text { Isolates } \\
\text { Detected as } \\
\text { MSSA }\end{array}$ & $\%$ \\
\hline Cefoxitin disc diffusion test (CDD) & 61 & 39 & 95 & 61 \\
\hline Oxacillin Disc Diffusion Method (ODD) & 50 & 32 & 106 & 68 \\
\hline Oxacillin screen agar (OSA) & 58 & 37 & 98 & 63 \\
\hline Oxacillin E strip (ES) & 58 & 37 & 98 & 63 \\
\hline ‘ChromID® MRSA SMART'(biomerieux) agar & 60 & 38 & 96 & 62 \\
\hline VITEK 2 automated system (biomerieux) & 61 & 39 & 95 & 61 \\
\hline Table 2. Number of MRSA and MSSA Isolates by Phenotypic Tests \\
\hline
\end{tabular}

\begin{tabular}{|c|c|c|c|c|}
\hline 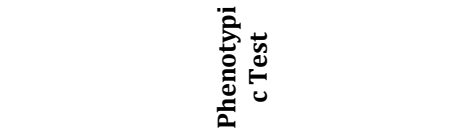 & 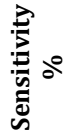 & 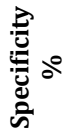 & $\frac{2}{2}$ & $\frac{a}{z}$ \\
\hline Cefoxitin disc diffusion test (CDD) & 100 & 100 & 100 & 100 \\
\hline The Oxacillin Disc Diffusion Method (ODD) & 81.96 & 97.89 & 96.15 & 89.42 \\
\hline Oxacillin screen agar (OSA) & 95.08 & 98.95 & 98.31 & 96.31 \\
\hline Oxacillin E strip (ES) & 98.36 & 95.79 & 93.75 & 98.91 \\
\hline “ChromID® MRSA SMART’(biomerieux) agar & 95.08 & 97.89 & 96.67 & 96.88 \\
\hline VITEK 2 automated system (biomerieux) & 100 & 100 & 100 & 100 \\
\hline \multicolumn{5}{|c|}{$\begin{array}{c}\text { Table 3. Sensitivity and Specificity, Positive Predictive Value and } \\
\text { Negative Predictive Value of Phenotypic Tests }\end{array}$} \\
\hline \multicolumn{5}{|c|}{ PPV- Positive Predictive Value. NPV- Negative Predictive Value } \\
\hline
\end{tabular}

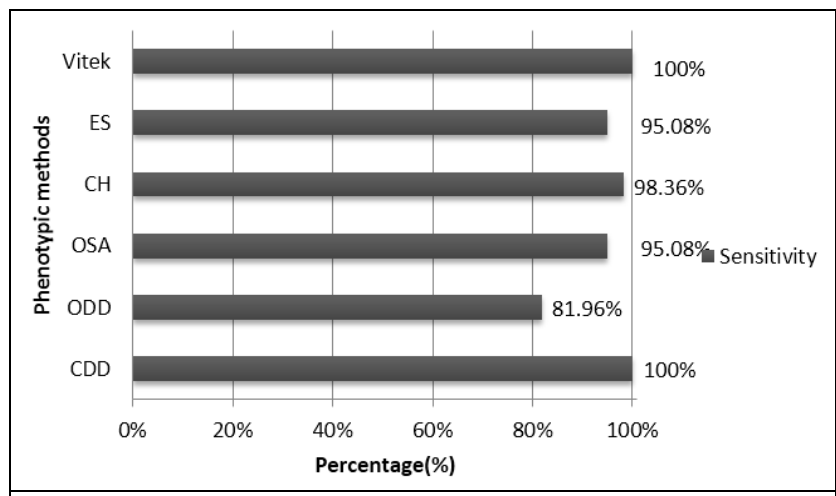

Figure 1. Graphical Comparison of Sensitivity of Phenotypic Methods CDD - Cefoxitin Disc Diffusion, ODD - Cefoxitin Disc Diffusion, OSA - Oxacillin Screen Agar, CH - Chromagar, ES - Oxacillin E-Strip Test, VITEK- VITEK- 2 System

\section{DISCUSSION}

For the past 50 years, Staphylococcus aureus has been a dynamic human pathogen that has gained the deepest respect 
of clinicians since the first report of MRSA infection in United States at a Boston city hospital.[10] Confirmation of the isolates is essential as the treatment differ in infection with MRSA to those with MSSA. Different methods were used for identification of MRSA strain. Confirmation requires detection of mecA gene by PCR, a costly test mostly inaccessible in majority of the centres. Therefore, cost effective reliable test method for detection of MRSA is needed in resource restricted set up.

We compared some commonly used 6 phenotypic tests in this study and analysed sensitivity, specificity, positive predictive value and negative predictive value. We found cefoxitin disc diffusion test as the most reliable phenotypic method with $100 \%$ sensitivity and specificity. These results are in agreement with the studies conducted by B Sasirekha et al, Pourmand MR et al and Abbas Farahari.[11,12,13] Thus the cefoxitin disk diffusion method is very suitable for detection of MRSA and the test can be an alternative to PCR for detection of MRSA in resource restrained settings. CLSI recommends cefoxitin disc diffusion as the surrogate test for methicillin resistance.[14]

Even though VITEK-2 automated system also showed $100 \%$ concordance with PCR, the comparative high cost limits its use in resource constrained settings. But the possibility of diagnosis of an MRSA infection in 4 hours and information about antibiotic susceptibility make this automated method very attractive for those clinical microbiology laboratories worldwide who can afford it.[15]

The sensitivity and specificity of oxacillin E-strip in our study was $95.08 \%$ and $97.89 \%$. Three isolates in our study showed discordant results for MIC of oxacillin and PCR, ie mecA positive, but oxacillin MIC $\leq 2 \mu \mathrm{g} / \mathrm{ml}$. This can be probably explained by the fact that not all Staphylococcus isolates express their mecA gene.[11,12]

Sensitivity and specificity of oxacillin screen agar were $95.08 \%$ and $98.95 \%$ respectively. A study by Reza et al reported $99.50 \%$ and $100 \%$ sensitivity and specificity for OSA. ${ }^{13}$ The presence of resistance in S. aureus isolates on an oxacillin screen agar plate generally means that the isolate is mecA positive. Occasionally, however, heteroresistant mecA positive strain is not detected due to low expression of resistance. Oxacillin agar screen generally does not detect borderline resistant strains, when studies have included strains whose resistance is heterogeneous the test has been shown to perform less well. [14]

ChromID ${ }^{\circ}$ MRSA SMART (biomerieux) agar is a ready to use chromogenic medium for the screening of methicillinresistant Staphylococcus aureus (MRSA). The sensitivity and specificity of this medium in our study was $98.36 \%$ and 95.79\% respectively. Another advantage of this selective chromogenic medium is that specimens can be directly inoculated. This permits early and direct detection of MRSA.[15]

The sensitivity and specificity of oxacillin disc diffusion (ODD) obtained in our study are $81.97 \%$ and $97.89 \%$ respectively. These findings are similar to many studies conducted elsewhere.[10,12] Misdiagnosis of such a large number of isolates is not acceptable because of the serious implications on the patient and the health care personnel. This results in worsening of the clinical condition, prolonged hospital stay, increased risk of spread to the healthcare personnel, spread to other patients and increased expenditure.

\section{Prevalence of MRSA in this Study}

Of the 156 staphylococcal isolates, methicillin sensitive strains predominated over methicillin resistant strains (60.89\% and 39.11\% respectively). The prevalence of MRSA in our study was $60.90 \%$. This finding is comparable to the findings of many studies conducted elsewhere in India.

\section{Susceptibility to Other Classes of Antibiotics}

In this study, among MRSA strains highest percentage of resistance was noted for ciprofloxacin (85.25\%). Also noticeably more resistance was noted for cotrimoxazole and erythromycin which are $77.05 \%$ and $67.22 \%$ respectively. $22.95 \%$ of MRSA strains were resistant to 3 drugs other than penicillin, $26.23 \%$ were resistant to 4 drugs other than penicillin and $13.11 \%$ strains were resistant to 5 drugs other than penicillin. In total $64.92 \%$ of the MRSA isolates were considered to be multidrug resistant.

\section{An Overview of Cost and Duration of Different Methods to Detect Methicillin Resistant Staphylococcus aureus.}

The time taken by routine culture and sensitivity methods for detection of MRSA is more as compared to PCR. Pillai et al ${ }^{16}$ made the following observations: "The time taken for diagnosing MRSA by conventional methods is $48-72 \mathrm{~h}$, which is more as compared to PCR which takes 18-24 h. The expenses and workload of single PCR exceed the demand of testing one clinical specimen for the presence of MRSA. But if the daily number of MRSA screening tests increases, the workload per PCR decreases and finally outweighs the expenses for molecular reagents. In comparison, the expenses due to the wrong diagnosis (Missing MRSA) will be far more than the cost of PCR.'

\section{CONCLUSIONS}

This study shows that cefoxitin disc diffusion test can be an alternative to mecA PCR in resource restrained setup. In a country like India where molecular methods are not feasible as a routine, cefoxitin disc diffusion test is a good surrogate marker of methicillin resistance. However, constant surveillance and breakpoint assessment should be carried out. However, always genotypic methods are the reference methods. Cost-effect analysis considering the expenditure caused by a misdiagnosis in one hand, and the benefit of timely detection and accuracy in other hand, prompts us to consider PCR for isolates from sterile body fluids or from intensive care units.

\section{REFERENCES}

[1] Barber M. Methicillin-resistant Staphylococci. Journal of Clinical Pathology 1961;14(4):385-93. 
[2] Chambers HF, DeLeo FR. Waves of resistance: Staphylococcus aureus in the antibiotic era. Nature Reviews Microbiology 2009;7(9):629-41.

[3] Stapelton PD, Taylor PW. Methicillin resistance in Staphylococcus aureus: mechanisms and modulation. Science Progress 2002;85(Pt 1):57-72.

[4] Mylotte JM, Tayara A. Staphylococcus aureus bacteremia: predictors of 30-day mortality in a large cohort. Clinical Infectious Diseases 2000;31(5):1170-4.

[5] Lynch JP 3rd. Hospital-acquired pneumonia: risk factors, microbiology and treatment. Chest 2001;119(Suppl 2):373S-84S.

[6] Blevins JS, Elasri MO, Allmendinger SD, et al. Role of sar$A$ in the pathogenesis of Staphylococcus aureus musculoskeletal infection. Infection and Immunity 2003;71(1):516-23.

[7] INSAR group (Indian Network for Surveillance of Antimicrobial Resistance). Methicillin resistant Staphylococcus aureus (MRSA) in India: Prevalence \& susceptibility pattern. Indian J Med Res 2013;137(2):363-9.

[8] Pai V, Rao VI, Rao SP. Prevalence and antimicrobial susceptibility pattern of Methicillin-resistant Staphylococcus aureus [MRSA] isolates at a tertiary care hospital in Mangalore, South India. Journal of Laboratory Physicians 2010;2(2):82-4.

[9] Tiwari HK, Sapkota D, Sen MR. High prevalence of multidrug-resistant MRSA in a tertiary care hospital of northern India. Infection and Drug Resistance 2008;1:57-61.
[10] Bhutia KO, Singh TS, Biswas S, et al. Evaluation of phenotypic with genotypic methods for species identification and detection of Methicillin resistance in Staphylococcus aureus. International Journal of Applied and Basic Medical Research 2012;2(2):84-91.

[11] Sasirekha B, Usha MS, Amruta AJ, et al. Evaluation and comparison of different phenotypic tests to detect Methicillin resistant Staphylococcus aureus and their biofilm production. International Journal of PharmTech Research 2012;4(2):532-41.

[12] Pourmand MR, Hassanzadeh S, Mashhadi R, et al. Comparison of four diagnostic methods for detection of Methicillin resistant Staphylococcus aureus. Iranian Journal of Microbiology 2014;6(5):341-4.

[13] Farahani A, Mohajeri P, Gholamine B, et al. Comparison of different phenotypic and genotypic methods for the detection of Methicillin-resistant Staphylococcus aureus. North American Journal of Medical Sciences 2013;5(11):637-40.

[14] Clinical Laboratory Standards Institute. Performance standards for, antimicrobial susceptibility testing. Twentieth informational supplement. CLSI document M100-S20. Wayne, PA: CLSI, 2006.

[15] Junkins AD, Lockhart SR, Heilmann KP, et al. BD phoenix and VITEK 2 detection of mecA-mediated resistance in Staphylococcus aureus with cefoxitin. Journal of Clinical Microbiology 2009;47(9):2879-82. 